\title{
Monoclonal Anti-idiotype Antibodies against Carbohydrate- associate Epitope for Anti-Cancer Vaccine Development
}

\author{
Gregory Lee*, Anthony P. Cheung, Bixia Ge, Mingang Zhu, Peter P. Li, Eric Hsu and Teng-Kai Huang \\ Andrology Lab, UBC Centre for Reproductive Health, Vancouver, Canada
}

\begin{abstract}
Background: RP215 monoclonal antibody (Mab) was shown to react specifically with the unique carbohydrateassociated epitope located in the variable regions of cancer cell-expressed immunoglobulins known as CA215. This Mab was shown to inhibit the growth of a variety of cancer cells in vitro or in vivo. Anti-idiotype (anti-id) Mabs against RP215 were generated and characterized for future development of epitope-specific anti-cancer vaccines in humans.

Results: Following successful immunization of rats with $F\left(a b^{\prime}\right)_{2}$ fragments of RP215, rat anti-id Mabs were established for biochemical and immunological characterization. Subsequent immunizations of mice with purified rat anti-id Mab revealed significant anti-anti-id antibody (Ab3) responses. In immunohistochemical studies, both Ab3 and RP215 reacted positively with various cancer cells lines and with comparable staining intensities. Both RP215 and Ab3 inhibited significantly the growth of cancer cells in vitro by means of TUNEL assay. To document that the RP215-specific epitope is carbohydrate-associated, the absence of RP215-epitope expressions upon culturing of cancer cells in serumfree medium was demonstrated. Experiments confirmed that the expression of RP215-specific epitope was dependent on the presence of carbohydrate precursors in culture media.
\end{abstract}

Conclusion: Judging from these observations, anti-id Mabs which carry the internal image of RP215-specific carbohydrate-associated epitope can be suitable candidates for anti-cancer vaccine development in humans.

Keywords: RP215; CA215; Anti-cancer vaccine; Apoptosis; Antiidiotype monoclonal antibody

Abbreviations: Ab3: Anti-anti-id Antibodies; Anti-id: Anti-idiotype; CA215: Pan Cancer Marker, Cancer Cell-expressed Immunoglobulin; ELISA: Enzyme-Linked Immunosorbent Assay; $\mathrm{F}\left(\mathrm{ab}^{\prime}\right)_{2}$ : Fragments obtained following pepsin digestion of immunoglobulin G; HRPRP215: Horse-Radish Peroxidase-labeled RP215; Mab: Monoclonal Antibody; TUNEL Assay: Terminal Deoxynucleotidyl Transferase dUTP nick end labeling (Assay for Apoptosis)

\section{Introduction}

It has been documented that immunoglobulins with undefined specificity are expressed by cancer cells in humans [1-10]. Recent studies have also indicated that these immunoglobulins are required for the growth and survival of cancer cells from different tissue origins in humans [4]. Hence, target-specific anti-cancer drugs can be developed, if we can identify unique biomarkers that can differentiate these cancer cell-expressed immunoglobulins from those originated from normal B cells.

RP215 is one of the three thousand monoclonal antibodies (Mabs) that we have generated against an ovarian cancer cell line, OC-3-VGH [11-13]. This antibody has been found to react specifically with a carbohydrate-associated epitope in the variable regions of cancer cell-expressed immunoglobulins, designated in general as CA215. More importantly, this specific epitope has not been found in normal human immunoglobulins $[11,12]$. Further studies have revealed that RP215 can inhibit tumor cell growth through its apoptotic or antiproliferative actions on cancer cells in vitro $[14,15]$. RP215 can also inhibit the growth of tumor cells in vivo as demonstrated by nude mouse experiments [16]. Through extensive molecular biological and immunohistochemical studies, RP215 has been shown to react with a broad spectrum of human cancer cells or tissues which express CA215 either on the cell surface and/or in secreted form [11]. Judging from these experimental observations, RP215 may be a suitable candidate for the development of antibody-based anti-cancer drugs for different types of human cancer that express CA215, if humanized forms of RP215 can be produced [16]. Since the carbohydrate-associated epitope of CA215 is recognized specifically by RP215, antibodies raised against the Fab (or variable) sequence of RP215 should mimic the internal image of the epitope. These antibodies are designated collectively as anti-id or Ab2. Subsequent immunizations with selected anti-id or the corresponding Fab fragment can potentially induce an immune response (Ab3) equivalent to that of RP215. Therefore, in principle, the anti-id can serve as effective vaccine to replace the original antigenic epitope and elicit RP215-like immune reactions against cancer cells in vivo which could result in neutralization of cancer cells $[14,15]$.

As a proof of concept, we have recently reported on the generation and preliminary characterization of polyclonal rabbit anti-id against RP215 [14,15]. However, it is impractical and ineffective to massproduce anti-id polyclonal antibodies without uniform properties for human applications as cancer vaccines. Therefore, rat anti-id Mabs of high affinity and specificity to RP215 may be a better starting point for human cancer vaccine development.

The molecular structure of RP215-specific carbohydrate-associated epitope has not yet been fully elucidated and highly purified CA215 bearing this unique epitope is still not available for further analysis.

*Corresponding author: Dr. Gregory Lee, Andrology Lab, UBC Centre for Reproductive Health, Rm D403, 4500 Oak Street, Vancouver, BC, V6H 3N1 Canada, Tel: +1 778322 4651; E-mail: andr@interchange.ubc.ca

Received October 25, 2010; Accepted October 30, 2010; Published Novembe 04, 2010

Citation: Lee G, Cheung AP, Ge B, Zhu M, Li PP, et al. (2010) Monoclonal Antiidiotype Antibodies against Carbohydrate-associate Epitope for Anti-Cancer Vaccine Development. J Vaccines Vaccin 1: 106. doi:10.4172/2157-7560.1000106

Copyright: (c) 2010 Lee G, et al. This is an open-access article distributed under the terms of the Creative Commons Attribution License, which permits unrestricted use, distribution, and reproduction in any medium, provided the original author and source are credited. 
So far, the anti-cancer efficacy of RP215 and its humanized forms have only been evaluated with cancer cells in vitro or with partially purified CA215 preparations. Therefore, in this study, efforts were made to generate and characterize rat anti-id Mabs against RP215 to further explore its potential for cancer vaccine development in humans [18-20]. Experiments were also performed by manipulating the cell culture conditions to further understand the immunogenic nature of the carbohydrate-associated epitope recognized by RP215.

\section{Materials and Methods}

\section{Antibodies}

RP215 monoclonal antibody (Mab) was generated by immunization of BALB/c mice with the extract of OC-3-VGH ovarian cancer cells followed by subsequent cell fusion, screening and characterization as described previously [13]. Through extensive biochemical and immunological studies, RP215 was shown to recognize a carbohydrate-associated epitope located in the variable regions of cancer cell-expressed immunoglobulins, known as CA215 [11,12]. Anti-idiotype (Anti-id) Mabs were generated in rats with $\mathrm{F}\left(\mathrm{ab} \mathrm{b}_{2}\right)_{2}$ fragments of RP215 as the immunogen. $\mathrm{F}\left(\mathrm{ab}^{\prime}\right)_{2}$ fragments of RP215 were produced by pepsin digestion as described previously $[14,15]$. Following three repeated immunizations at monthly intervals, cell fusion between the spleen cells of immunized rats and rat myeloma cells (Y3) was performed [20-22]. More than 1000 hybrid clones were initially screened. Finally, nine hybrid cell lines were established and shown to secrete anti-id Mabs which exhibited high specificity and affinity to the $F(a b ')_{2}$ fragments of RP215, but not to those of normal mouse IgG. The affinity of rat monoclonal antibodies to RP215 was ranked by and established method using ELISA with microwells coated with $\mathrm{F}(\mathrm{ab})_{2}$ of RP215 [23]. One of the rat anti-id Mabs designated as R15H6 was shown to reveal the highest binding affinity to RP215 and further purified from large-scale, cell-culturedshed media by immunoaffinity chromatography with RP215-linked affinity gel as described previously [13].

Anti-anti-id (Ab3) sera were generated in BALB/c mice to induce $A b 3$ responses. Typical immunizations were performed with $F(a b ')_{2}$ fragments of R15H6 monoclonal antibody as the immunogen. After the fourth immunization, Ab3 sera from three immunized mice were collected separately for further characterization by various biochemical and immunological methods.

\section{Immunoassays}

The specificity of rat anti-id Mabs was determined by enzymelinked immunosorbent assays (EIA) targeting at RP215 or $\mathrm{F}\left(\mathrm{ab}^{\prime}\right)_{2}$ fragments of RP215. In the former, rat anti-id Mabs in hybridoma culture supernatants were incubated with microwells coated with RP215 and HRP-labeled RP215 in a sandwich enzyme immunoassay for one hour at $37^{\circ} \mathrm{C}$ as reported previously [17]. In the latter, the binding assay was performed between rat anti-id Mabs and microwells coated with $\mathrm{F}(\mathrm{ab})^{\prime}$, fragments of RP215 before adding alkaline phosphatase-labeled goat anti-rat $\operatorname{IgG}$ as the secondary antibodies in a typical ELISA [11]. The relative binding affinity of RP215 to CA215 or to R15H6 Mab was also studied with ELISA using wells coated with affinity purified CA215 or R15H6 Mabs, respectively. Appropriate positive and negative controls were included in all the immunoassays to ensure the validity of the assay results.

\section{Immunohistochemical staining studies and Western blot assay}

The ability of RP215 and mouse anti rat anti-id (Ab3) sera to react with human cancer cells from more than twenty different cell lines was assessed by immunohistochemical staining and Western blot assay. Detailed procedures of immunohistochemical staining studies using the avidin-biotin complex $(\mathrm{ABC})$ method (Vector Lab, Burlingame, $\mathrm{Ca}$ ) were described previously [16]. For studies with Ab3 sera, both rat $\operatorname{IgG}$ and human $\operatorname{IgG}$ of $10 \mu \mathrm{g} / \mathrm{mL}$ were included in the assay during the first step of incubation to eliminate any possible cross-reactivity between rat $\operatorname{Ig} G$ and human $\lg G$ during the staining of cancer cells. Western blot assay was performed using SDS-PAGE (with or without reducing agent) for OC3-VGH cell extract. This was followed by incubation of the blot-transferred membrane with primary antibodies containing RP215 or Ab3 in comparative studies. The protein bands were detected with the alkaline phosphatase-labeled goat anti-mouse $\operatorname{IgG}$ as the secondary antibodies before adding the substrate (BioRad, Richmond, Ca) for color development.

\section{Study of apoptosis by TUNEL assay}

The TUNEL assay was performed to assess apoptosis of cultured cancer cells in vitro upon incubation with RP215 or Ab3 sera. Details of the TUNEL assay were presented previously $[14,15]$. Briefly, cells from cancer cell lines were cultured in RPMI-1640 containing $10 \%$ fetal calf serum. RP215 $(10 \mu \mathrm{g} / \mathrm{mL})$ or Ab3 sera (1:1000 dilution) were added to the cultured cancer cells for 24,48 and 72 hours of incubation. At the end of incubation, cells were harvested and TUNEL assay was performed using In Situ Cell Death Detection Kit, POD (Roche Diagnostics, Canada) according to instructions provided in the package insert. Normal mouse sera of the same concentrations or dilutions served as negative controls. Percent increases in apoptosis of treated cells were compared with those of negative controls. Analysis of variance was used to compare experimental results and statistical significance was defined at a $P$ value of $<0.05$.

\section{Culture of cancer cells in serum-free and serum-supplemented media}

Experiments were performed to determine if carbohydrates or their precursors were required for the expression of RP215-specific epitope found in cancer cell-expressed CA215. OC-3-VGH ovarian cancer cells were cultured separately in serum-free (FS293 serum free medium, Invitrogen) and serum-containing media (10\% bovine calf serum in RPMI-1640) for 48 hours. CA215 in each culture shed medium was assayed using RP215-based ELISA kit and Western blot assay to determine the relative activity of RP215-specific epitope. The concentration of human $\operatorname{IgG}$ in each shed medium was also determined separately by an ELISA kit with goat anti-human IgGcoated microwells and paired with HRP-labeled anti-human IgG-FC Mab for a one hour assay [11]. The OC-3-VGH ovarian cancer cell line was originally established in 1986 by the Dept. OBS/GYN at the Veterans General Hospital, Taipei, Taiwan and maintained in RPMI-1640 medium containing $10 \%$ fetal calf serum [13]. The other cancer cell lines were obtained from ATCC (American Type Culture Collection, Rockville, MD). FS293 (kidney) cell line was obtained from Invitrogen (Burlington, ON, Canada). These cell lines were maintained according to the suppliers' instructions. Serum-supplemented and serum-free media were obtained from Sigma (St. Louis, MO) and Invitrogen, respectively.

\section{Results}

\section{Generation and characterization of rat anti-id mabs}

Rat hybridomas were generated after cell fusion and screening for the secretion of Mabs against $F(a b$ ') , fragments of RP215. All nine established anti-id Mabs obtained from the cell supernatants were 
Citation: Lee G, Cheung AP, Ge B, Zhu M, Li PP, et al. (2010) Monoclonal Anti-idiotype Antibodies against Carbohydrate-associate Epitope for AntiCancer Vaccine Development. J Vaccines Vaccin 1: 106. doi:10.4172/2157-7560.1000106

Page 3 of 7

shown to react with $\mathrm{F}(\mathrm{ab})_{2}$, fragments of RP215 coated on microwells in a dose dependent manner. The results of the typical binding assays represented by the three anti-id Mabs, R3E4, R3E7 and R15H6 are presented in Figure $1 \mathrm{~A}$. In separate experiments, rat anti-id Mabs were treated as the surrogate antigen and compared with CA215 in a RP215-based ELISA. However, R3E4 showed no binding activity when determined by this sandwich immunoassay. The corresponding assay results are presented in Figure 1B for comparative purpose. Among these Mabs, R15H6 was shown to have the highest affinity $\left(K_{d} \leq 1\right.$ $\mathrm{nM}$ ) and specificity to $\mathrm{F}(\mathrm{ab})_{2}$, fragments of RP215 [23]. In a separate experiment, R15H6 Mab was shown to inhibit the binding between OC-3-VGH cancer cells coated on microwells and HRP-labeled RP215 Mab in a dose-dependent manner (data not shown), similar to that observed for polyclonal rabbit anti-id of RP215 antibodies [15].

\section{Characterization of Ab3 sera}

The purified rat anti-id Mab, R15H6, was used as the immunogens to immunize BALB/c mice. Mouse anti rat anti-id (Ab3) sera were generated following successive immunization with $\left.\mathrm{F}(\mathrm{ab})_{2}\right)_{2}$ of R15H6 Mab. Using ELISA with CA215-coated microwells, Ab3 sera were found to react with CA215 in a dose-dependent manner, comparable to that of RP215 (Figure 2A).

Western blot assay was employed to determine the molecular weight of the detected protein bands recognized by Ab3 sera and RP215 under the reducing and non-reducing conditions of SDS-PAGE in OC-3-VGH cell extracts [11]. As shown in Figure 2B, similar broad protein bands of $160 \mathrm{kDa}$ were observed for RP215 or Ab3 sera under the non-reducing condition. In contrast, protein bands of $60 \mathrm{kDa}$ were observed under the reducing condition when RP215 or Ab3 sera were used as the probe. The equivalence between RP215 and Ab3 sera in terms of these respective binding specificity to CA215 was demonstrated by this experiment.

\section{Immunohistochemical studies}

Both RP215 and Ab3 sera were used as the probe in immunohistochemical staining studies of cancer cell lines of various tissue origins in humans [16]. Among these cell lines are those of the ovary (OC-3-VGH and SKOV-3), cervix (C-33A) and lung (A549). Both RP215 $(1 \mu \mathrm{g} / \mathrm{mL})$ and Ab3 sera (1:500 dilution) showed prominent staining intensities. In contrast, normal mouse serum gave no staining to the cancer cells. Typical results of this comparative study are summarized in Figure 3A.

As many as twenty other human cancer cell lines of different tissue origins from ATCC were also employed for comparative immunohistochemical staining studies. The following cell lines showed comparable staining intensities for both RP215 and Ab3 sera: breast cancer cell lines MCF7 (HTB-22), MDA-435, MDA-231 and T47D (HTB-133); placenta cancer cell line BeWo (CCL-98); cervical cancer cell lines C-33A and ME180 (HTB-33); colon cancer cell lines Sw-48 (CCL-231), HCT-115, HCT-116, HT-441 and HT-29 (HTB 38); brain cancer cell lines Neura2A, SH-SY5Y (CRL-2266) and U-87MG (HTB-14); liver cancer cell lines HepG2 (HB-8065), Hep-2 and Hep-3B (HB-8064); kidney cancer cell line FS293 (Invitrogen); lung cancer cell lines A549 (CCL-185), Calu-6 (HTB-56), MRC-5 (CCL-171) and WI-38 (CCL-75); melanoma cell line MMAN; ovarian cancer cell lines SKOV-3 (HTB-77), OC-3-VGH and OVCAR-3 (HTB-161); prostate cancer cell lines DU-145 (HTB-81) and PC-3 (CRL-1435). However, the following cancer cell lines showed weak or no staining for both RP215 and Ab3 sera under the same conditions: Hela (CCL-2 cervix), SK-MEL-3 (HTB-69, melanoma),

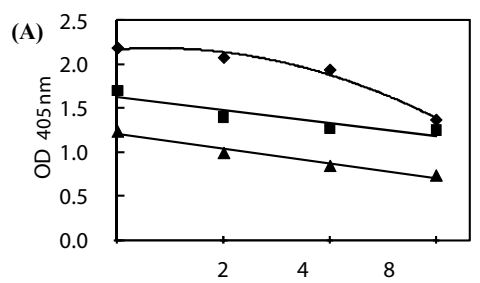

Dilution Factor (Log Scale)

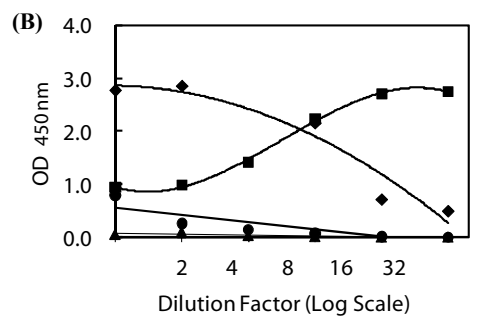

Figure 1: (A) Dose-dependent binding between rat anti-id Mabs in cell supernatants and microwell-coated RP215 F(ab') on ELISA described in the text. Alkaline phosphatase-labeled goat anti-rat IgG was used as the secondary antibody for signal detection at $405 \mathrm{~nm} ;(\boldsymbol{\Delta}),(\bullet)$ and $(\boldsymbol{\bullet})$ denote rat anti-id mabs, R3E4, R3E7 and R15H6 in cell culture medium (RPMI1640 plus $10 \%$ fetal calf serum), respectively (estimated $\mathrm{K}$ for $\mathrm{R} 15 \mathrm{H} 6$ binding to RP215-F(ab') $\left.)_{2}: \leq 1 \times 10^{-9} \mathrm{M}\right)$. Normal rat IgG $(10 \mu \mathrm{g} / \mathrm{ml}$ ) in cell culture medium (RPMI1640 plus 10\% fetal calf serum was used as the negative control. Data presented are those substrated from the negative control $(\mathrm{OD} \leqq 0.1)$. (B) Dose-

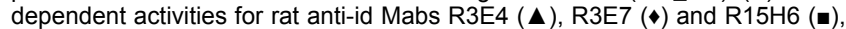
as well as CA215 $(\bullet)$ in RPMI1640 cell culture medium (10\% fetal calf serum) on RP215-based sandwich EIA as described in the text. Normal rat lgG $(10 \mu \mathrm{g} /$ $\mathrm{ml}$ ) in the same culture medium served as the negative control. Data presented are those subtracted from the negative control $(\mathrm{OD} \leqq 0.1)$.

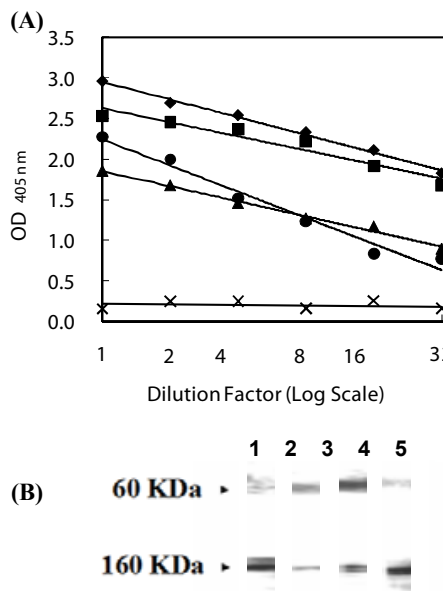

Figure 2: (A) Dose-dependent binding between Ab3 (anti-anti-id) sera from three immunized mice or RP215 and microwell-coated CA215. Alkaline phosphatase-labeled goat anti-mouse $\lg$ was used as the secondary antibody for signal detection at $405 \mathrm{~nm}$. ( $\bullet),(\boldsymbol{\bullet})$ and $(\boldsymbol{\Delta})$ denote Ab3 sera from three BALB/c mice (designated as L, $R$ and I) immunized with rat anti-id Mab, respectively (initial dilution $1: 200) .(\bullet)$ and $(x)$ denote RP215 and normal mouse $\mathrm{lgG}$ (initial concentration $=1 \mu \mathrm{g} / \mathrm{mL}$ ), respectively. (B) Western blot assay to compare molecular weight of the protein bands detected in OC-3VGH cancer cell extract using RP215 or Ab3 sera as the probe with reducing and non-reducing SDS-PAGE. Molecular markers of $60 \mathrm{KDa}$ and $160 \mathrm{KDa}$ were shown on the left. From left to right: Lane 1: RP215; Lanes 2, 3 and 4 are Ab3 sera from three immunized mice designated as $I, R$ and $L$, respectively (concentration $=1: 10,000$ dilution); Lane 5 is negative control with normal mouse sera of the same dilution.

HEL (lymphoma) and K562 (CCL-243, leukemia). The results of these immunohistochemical staining studies are summarized in Table 1. 


\section{TUNEL assay for studies of cellular apoptosis}

By means of TUNEL assay, it could be shown that both RP215 and Ab3 sera induced significant degrees of apoptosis to culture cancer cells. Both OC-3-VGH ovarian cancer cells and C-33A cervical cancer cells were employed for this study, as shown in Figure 3B. Upon incubation of cultured OC-3-VGH cancer cells with $10 \mu \mathrm{g} / \mathrm{ml}$ of RP215 or $\mathrm{Ab} 3$ serum in 1:500 dilution for $24 \mathrm{hrs}, 50-80 \%$ of the cultured cells underwent apoptosis as compared to the negative control of about $15 \%(\mathrm{P}<0.05)$. A similar degree of apoptosis can be induced upon incubation of $A b 3$ serum of the same dilution for 72 hrs in cultured C-33A cancer cells when compared with that of RP215 under the same conditions (Figure 3B, lanes IV and V).

\section{Comparison of CA215 in shed media of cultured cancer cells in serum-free and serum-supplemented conditions}

CA215 in shed media was recovered under both serum-free and serum-supplemented conditions and assayed separately for the relative concentrations of RP215-specific epitope as well as those of human $\operatorname{IgG}$ for cultured cells from OC-3-VGH and C-33A. For both cell types, RP215-based sandwich enzyme immunoassay showed dose-dependent binding activities to CA215 in shed media under serum-supplemented but not serum-free conditions (Figure 4A). In contrast, immunoassays of human $\operatorname{IgG}$ in both serum-free and serum-supplemented shed media of cultured cancer cells revealed comparable dose-dependent activities when probed with anti-human IgG-Fc Mab (Figure 4B).

\begin{tabular}{|c|c|c|c|c|}
\hline Cancer Types & Cell Lines & ATCC Number & RP215 & Ab3 $^{a}$ \\
\hline \multirow[t]{3}{*}{ Brain } & Neura2A & & $\pm^{\mathrm{b}}$ & \pm \\
\hline & SH-SY5Y & CRL-2266 & + & + \\
\hline & U-87MG & HTB-14 & + & + \\
\hline \multirow[t]{4}{*}{ Breast } & MCF7 & HTB-22 & \pm & \pm \\
\hline & MDA-MB-231 & HTB-26 & + & + \\
\hline & MDA-435 & & + & + \\
\hline & T-47D & HTB-133 & \pm & \pm \\
\hline \multirow[t]{3}{*}{ Cervical } & C-33A & HTB-31 & + & + \\
\hline & Hela & CCL-2 & - & - \\
\hline & ME-180 & HTB-33 & + & + \\
\hline \multirow[t]{5}{*}{ Colon } & HCT 115 & & + & + \\
\hline & HCT 116 & CCL-247 & + & + \\
\hline & HT-29 & HTB-38 & + & + \\
\hline & HT-441 & & + & + \\
\hline & SW-48 & CCL-231 & + & \pm \\
\hline Kidney & FS293 & & + & + \\
\hline Leukemia & K562 & CCL-243 & - & - \\
\hline \multirow[t]{3}{*}{ Liver } & Hep-2 & & + & + \\
\hline & HepG2 & HB-8065 & \pm & \pm \\
\hline & Нер3B & HB-8064 & + & + \\
\hline \multirow[t]{4}{*}{ Lung } & A549 & CCL-185 & + & + \\
\hline & Calu-6 & HTB-56 & + & + \\
\hline & MRC-5 & CCL-171 & + & + \\
\hline & WI-38 & CCL-75 & + & + \\
\hline Lymphoma & HEL & & - & - \\
\hline \multirow[t]{2}{*}{ Melanoma } & MMAN & & + & + \\
\hline & SK-Mel-3 & HTB-69 & - & - \\
\hline \multirow[t]{3}{*}{ Ovary } & OC-3-VGH & & + & + \\
\hline & OVCAR-3 & HTB-161 & + & + \\
\hline & SK-OV-3 & HTB-77 & + & + \\
\hline Placenta & BeWo & CCL-98 & \pm & \pm \\
\hline \multirow[t]{2}{*}{ Prostate } & DU145 & HTB-81 & \pm & \pm \\
\hline & PC-3 & CRL-1435 & \pm & \pm \\
\hline
\end{tabular}

ab3: Antisera raised rat anti-RP215-idiotype monoclonal antibody

bStaining intensities follow the order of - (negative), \pm (borderline) and + (positive) ND: not determined

Table 1: Immunohistochemical Staining Results of Various Cancer Cell Lines with RP215 and Other Relevant Antibodies.

\section{(A)}

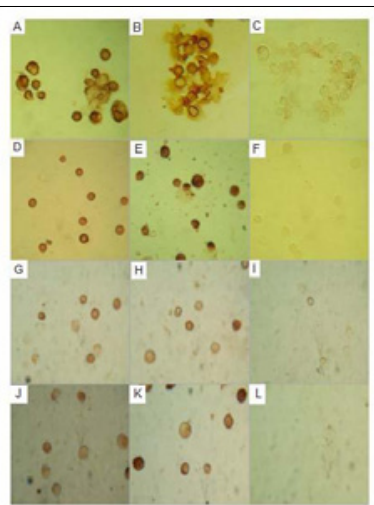

(B) 100

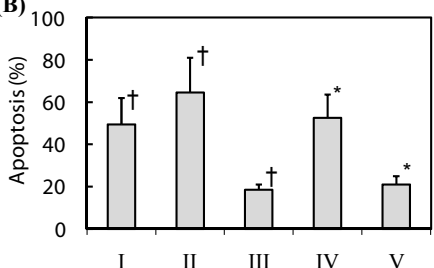

Figure 3: (A) Immunohistochemical staining (IHC) assay to compare relative staining intensities of different cancer cell lines probed with RP215 Mab or Ab3 sera. From left to right and top to bottom: Panels $A, B$ and $C$ are $\mathrm{IHC}$ staining

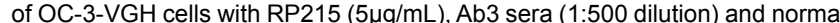
mouse serum, respectively. Panel D, E and $F$ are those of C-33A with RP215, Ab3 sera and normal mouse serum, respectively. Panel G, $\mathrm{H}$ and $\mathrm{I}$ are those of A549 cells with RP215, Ab3 sera and normal mouse serum, respectively. Panels J, K and L are those of SKOV-3 cells with RP215, Ab3 sera and normal mouse serum, respectively. (B) Percent increases in apoptosis of cancer cells after incubation with RP215 $(10 \mu \mathrm{g} / \mathrm{mL})$ and Ab3 sera (1:500 initial dilution) on TUNEL assay. Lanes I, II and III are percentage increases in OC-3-VGH cancer cell apoptosis after 24 hrs incubation with RP215, Ab3 sera and norma mouse serum of the same dilution (negative control), respectively. Lane IV and $\mathrm{V}$ are $\mathrm{C}-33 \mathrm{~A}$ cancer cell apoptosis after $72 \mathrm{hrs}$ incubation with Ab3 and norma mouse serum of the same dilution, respectively. Each column represents the mean values \pm S.D. of 4 determinations; $\dagger$ and ${ }^{*}$ were relative to negative controls with $p<0.05$.

Western blot assay was employed to compare the relative activities of RP215-specific epitope as well as that of human $\operatorname{IgG}$ between serum-free and serum-supplemented shed media of cultured cancer cells (Figure 4C). When RP215 was used as the probe, only serum-supplemented shed media revealed protein bands of $60 \mathrm{kDa}$, whereas no protein bands were observed for those of serum-free shed media for both OC-3-VGH and C-33A cells. In contrast, when an anti-human IgG-Fc Mab was used as the probe in Western blot assay, both serum-free and serum-supplemented media revealed the same protein bands of $60 \mathrm{kDa}$ which corresponded to those of heavy chains of human immunoglobulins. Furthermore, immunohistochemical studies revealed that RP215 failed to react with cancer cells of several cell lines after they were cultured in serum-free media (data not shown).

\section{Discussion}

In this communication, efforts were made to generate and characterize rat anti-id Mabs against RP215-specific epitope. Ab3 responses from the immunization of anti-id Mabs in mice were evaluated to serve as the model for future anti-cancer vaccine development in humans. The expression of RP215-specific epitope was assessed under different culture conditions to further document the carbohydrate association of this unique epitope.

Our previous studies have suggested that RP215 Mab recognizes a unique carbohydrate-associated epitope in the Fab regions of 
immunoglobulins expressed by the majority of cancer cells in humans [11]. This unique epitope, however, is not detected in normal human immunoglobulins [11]. It has also been suggested previously that immunoglobulin expression by cancer cells are widespread and are required for cancer cell survival and proliferation $[4,14,15]$. The expressed immunoglobulins could either be on the cancer cell surface or in secreted forms. Subsequently, RP215 has also been shown to inhibit the growth of cancer cells in vitro by apoptosis assay $[14,15]$ and by nude mouse experiments in vivo [16].

We have demonstrated previously that polyclonal rabbit antiid generated against RP215-F(ab') ${ }_{2}$ could in principle serve as the internal image of the RP215-specific carbohydrate-associated epitope. This is corroborated by the fact that Ab3 generated against rabbit anti-id exhibits similar functional properties as those of RP215 on apoptosis and anti-proliferation assays. However, for practical reasons, polyclonal rabbit anti-id is not an ideal choice for human cancer vaccine development that requires highly homogenous and specific properties. Therefore, in this study, efforts were made to generate and characterize rat anti-id Mabs against RP215-F(ab') with various biochemical and immunological methods. Each of the rat anti-id Mabs is a singly-defined molecule and can be mass produced in vitro or in vivo. Subsequent immunization with these highly specific anti-id Mabs can then induce targeted $\mathrm{Ab} 3$ responses to neutralize cancer cells better than the rabbit polyclonal counterparts [14]. This approach could, in principle, bypass the requirements for structural identification and synthesis of this unique carbohydrateassociated epitope linked to heterogeneous cancer cell-expressed immunoglobulins.

Results of the present study indicate that most of the generated rat anti-id Mabs bear the properties of the internal image of RP215specific epitope. This assumption is based on the differential results of RP215-based immunobinding assay (Figure 1A) and sandwich enzyme immunoassay (Figure 1B). Among these Mabs, R3E4 showed good binding to RP215, yet failed to produce any significant activity in RP215-based sandwich enzyme immunoassay. The other Mabs can be used as the surrogate antigen of CA215 in RP215-based enzyme immunoassay. Among these, R15H6 Mab revealed the highest affinity to RP215 and was selected as the immunogen to generate Ab3 responses in immunized mice. Ab3 sera were found to have binding to well-coated CA215, comparable to that of RP215. By using Western blot assay, both Ab3 sera and RP215 were found to recognize the same protein bands in the cancer cell extract of $60 \mathrm{KDa}$ and $160 \mathrm{KDa}$, depending on the presence or absence of reducing agents (Figure 2B). In addition, both RP215 and Ab3 sera gave positive staining to various cancer cells with comparable staining intensities in immunohistochemical studies (Figure 3A). The results further confirm the expression of CA215 on the majority of cancer cell surface [4]. This observation strongly supports the development of rat antiid Mab as anti-cancer vaccine with multi-indication in humans. Furthermore, by using TUNEL assay, both RP215 and Ab3 sera were shown to induce apoptosis of two different types of cultured cancer cells in vitro (Figure 3B). This observation indicates that Ab3 sera can also inhibit the growth of cancer cells in vitro through the mechanism of apoptosis similar to that observed for RP215 [14,15].

The expression of RP215-specific carbohydrate-associated epitope in CA215 from cancer cells was compared under different cell culture conditions. Following 48 hrs culture of cancer cells in the serum-free medium, RP215-specific epitope was undetectable in the shed medium (Figure 4A). In contrast, the $\operatorname{IgG}$ content in the serum-free shed medium remained the same as that in serum- supplemented shed media (Figure 4B). The result of Western blot assay further confirmed the absence of RP215-specific epitope in CA215 recovered from serum-free media (Figure 4C). On the contrary, the expression of $\lg G$ by cancer cells at $48 \mathrm{hrs}$ remained detectable and was independent of the cell culture conditions (Figure 4C). These experimental observations clearly indicate that post-translational modification of CA215 is essential to generate RP215-specific epitope and requires carbohydrate precursors present in serumsupplemented media, but not in serum-free media.

Cancer treatment vaccines are designed to treat cancer by stimulating the immune system to recognize and attack cancer cells [24-27]. Although the US FDA has only approved a cancer treatment vaccine, numerous anti-cancer treatment vaccines are being developed against many types of cancer and are being tested in clinical trials [28-30]. Cancer treatment with anti-idiotype antibodies has been reported previously with limited efficacy [28], especially in the case of B cell lymphoma [32]. For melanoma, anti-id Mabs mimicking HMW-

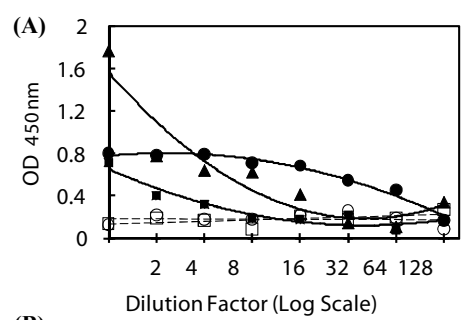

(B)

Dilution Factor (Log Scale)

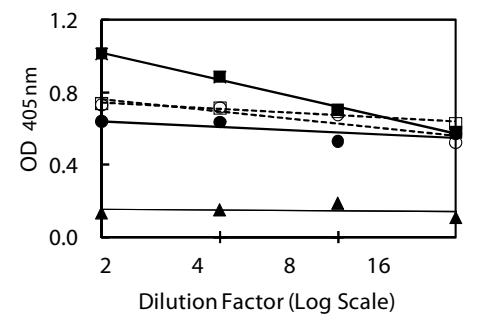

(C)

$\begin{array}{llllllll}1 & 2 & 3 & 4 & 5 & 6 & 7 & 8\end{array}$

$60 \mathrm{KDa}+-\quad-$

$60 \mathrm{KDa}$.

Figure 4: (A) RP215-specific epitope activities of the shed media from OC 3 -VGH and C-33A cells cultured in serum-free and serum supplemented media for 48 hrs by using RP215/RP215-HRP-based enzyme immunoassays. Absorbance at $450 \mathrm{~nm}$ was plotted against serial dilutions of the shed media; $(\bullet)$ and $(\bullet)$ represent activities in serum-supplemented media for OC-3-VGH and C-33A cells, respectively;( $\square)$ and $(\circ)$ represent corresponding activities in serum-free media; $(\boldsymbol{\Delta})$ represents the initial activities of $10 \mathrm{AU} / \mathrm{mL}$ CA215. Fresh RPMI-1640 culture medium containing 10\% fetal calf serum was used as the negative control $(O D \leq 0.1)$; Data presented have been substrated from the negative control; (B) Relative human IgG concentrations in shed culture media of OC-3-VGH and C-33A cancer cells under serum-supplemented and serum-free conditions for $48 \mathrm{hrs}$ using the anti-human IgG-Fc Mab as the probe on ELISA. ( $\bullet$ ) and $(\bullet)$ represent human IgG activities in serumsupplemented media for OC-3-VGH and C-33A cells, respectively; ( $\square$ ) and (०) represent corresponding activities in serum-free media. Fresh cell culture medium (RPMI plus $10 \%$ bovine calf serum) was used as the negative control $(\boldsymbol{\Delta})$. (C) Western blot assay to detect protein bands from cultured cancer cells under serum-free and serum-supplemented conditions. Upper panels are protein bands of $60 \mathrm{KDa}$ detected under reducing SDS-PAGE using RP215 as the probe. From left to right: Lane 1: OC-3-VGH cells (serum plus), Lane 2: OC-3-VGH cells (serum-free), Lane 3: C-33A cells (serum plus), Lane 4: C-33A cells (serum-free), Lane 5: FS293 cells (serum plus), Lane 6: FS293 cells (serum-free), Lane 7: RPMI medium control, Lane 8: serum-free medium control. Lower panels are protein bands of $60 \mathrm{KDa}$ detected under reducing SDS-PAGE using anti-human IgG-Fc Mab as the probe. From left to right: samples from Lane 1 to 8 are identical to those described in the upper panel. 
Citation: Lee G, Cheung AP, Ge B, Zhu M, Li PP, et al. (2010) Monoclonal Anti-idiotype Antibodies against Carbohydrate-associate Epitope for AntiCancer Vaccine Development. J Vaccines Vaccin 1: 106. doi:10.4172/2157-7560.1000106

MAA (high molecular weight melanoma-associated antigen) have been attempted in small multi-cancer trials [31-34]. Vaccination can elicit strong and specific humoral responses to relevant tumor-associated antigens [34] but clinical implications of these results remain to be fully elucidated [34]. In the case of colorectal cancer, anti-id vaccines for colorectal carcinoma-associated antigens, such as CEA, were also developed. Encouraging preliminary results from pilot studies still await further confirmation [35]. The anti-id Mab mimicking the tumor antigen, CA125 (abagovomab) has been recently reported in a limited number of clinical trials for ovarian cancer patients. A CA125-specific humoral response was elicited in nearly half of the patients and a correlation to patients' survival was suggested [36].

Judging from numerous on-going clinical studies, the RP215specific carbohydrate-associated epitope which is highly associated with cancer cell-expressed immunolglobulins appears to be a suitable candidate cancer vaccine development. The anti-id monoclonal antibodies which mimic the internal image of this unique epitope could also serve as the alternative for cancer vaccine development as demonstrated by the in vitro studies presented in this report. Moreover, the carbohydrate-associated epitope-based anti-cancer vaccines can potentially target cancer cells of many different human tissue origins. Additional in vivo preclinical studies are in progress to demonstrate the efficacy of the anti-id Mab-based anti-cancer vaccines.

\section{Conclusion}

In summary, RP215-specific carbohydrate-associated epitope can be used to generate specific anti-id antibodies with potential anticancer vaccine properties to induce $A b 3$ response in animal models and in humans. Ab3 sera are as effective as RP215 Mab in inhibiting cancer cell growth in vitro [20-22]. Therefore, anti-id Mab against RP215 is a potential candidate for anti-cancer vaccine development in humans.

\section{Acknowledgements}

This research work was supported in parts by NSERC IRAP (\#70395) and NSERC IRDF (\#360647) programs. The technical supports and scientific advices from the following individuals are acknowledged: Jerry Zheng, Wei Zhang, $\mathrm{Yu} \mathrm{Li}$ and Hsiao Yun Wang.

\section{References}

1. Liu HD, Zheng H, Li M, Hu DS, Tang M, et al. (2007) Upregulated expression of kappa light chain by Esptein-Barr virus encoded latent membrane protein 1 in nasopharyngeal carcinoma cells via NF-kB and AP-1 pathways. Cell Signal 19: 419-427.

2. Zheng H, Li M, Ren W, Zeng L, Liu HD, et al. (2007) Expression and secretion of immunoglobulin alpha heavy chain with diverse VDJ recombinations by human epithelial cancer cells. Mol Immunol 44: 2221-2227.

3. Li M, Feng DY, Ren W, Zheng L, Zheng, et al. (2007) Expression of immunoglobulin kappa light chain constant region in abnormal human cervical epithelial cells. Int J Biochem Cell Biol 36: 2250-2257.

4. Qiu X, Zhu X, Zhang L, Mao Y, Zhang J, et al. (2003) Human epithelial cancers secrete immunoglobulin $\mathrm{G}$ with unidentified specificity to promote growth and survival of tumor cells. Cancer Res 63: 6488-6495.

5. Kimoto $Y$ (1998) Expression of heavy-chain constant region of immunoglobulin and T-cell receptor gene transcripts in human non-hematopoietic tumor cell lines. Genes Chromosomes Cancer 22: 83-86.

6. Zhu X, Li C, Sun X, Mao Y, Li G, et al. (2008) Immunoglobulin mRNA and protein expression in human oral epithelial tumor cells. Appl Immunohistochem Mol Morphol 16: 232-238.

7. Babbage G, Ottensmeier CH, Blaydes J, Stevenson FK, Sahota SS (2006) Immunoglobulin heavy chain locus events and expression of activation-induced cytidine deaminase in epithelial breast cancer cell lines. Cancer Res 66: 39964000 .
8. Geng LY, Shi ZZ, Dong Q, Cai XH, Zhang YM et al. (2007) Expression of SNC73, a transcript of the immunoglobulin $\alpha-1$ gene, in human epithelial carcinomas. World J Gastroenterol 13: 2305-2311.

9. Chen Z, Gu J (2007) Immunoglobulin G expression in carcinomas and cancer cell lines. The FASEB J 21: 2931-2938.

10. Forconi F, Sahota SS, Raspadori D, Mockridge CI, Lauria F, et al. (2001) Tumo cells of hairy cell leukemia express multiple clonally related immunoglobulin isotypes via RNA splicing. Blood 98: 1174-1181.

11. Lee G, Laflamme E, Chien $\mathrm{CH}$, Ting $\mathrm{HH}$ (2008) Molecular identity of a pan cancer marker, CA215. Cancer Biol Ther 7: 2007-2014.

12. Lee G, Ge B (2009) Cancer cell expressions of immunoglobulin heavy chains with unique carbohydrate-associated biomarker. Cancer Biomark 5: 177-188.

13. Lee CYG, Chen KW, Sheu FS, Tsang A, Chao KC, et al. (1992) Studies of a tumor associated antigen, COX-1, recognized by a monoclonal antibody. Cancer Immunol Immunother 35: 19-26.

14. Lee G, Ge B (2010) Growth inhibition of tumor cells in vitro by using monoclonal antibodies against gonadotropin-releasing hormone receptor. Cancer Immunol Immunother 59: 1011-1019.

15. Lee G, Ge B (2010) Inhibition of in vitro tumor cell growth by RP215 monoclonal antibody and antibodies raised against its anti-idiotype antibodies. Cancer Immunol Immunother 59: 1347-1356.

16. Lee G, Chu RA, Ting HH (2009) Preclinical assessment of anti-cancer drugs by using RP215 monoclonal antibody. Cancer Biol Ther 8: 161-166.

17. Lee G (2009) Cancer cell-expressed immunoglobulins: CA215 as a pan cancer marker and its diagnostic applications. Cancer Biomark 5: 137-142.

18. Lowder JN, Meeker TC, Campbell M, Garcia CF, Garlow J, et al. (1987) Studies on B lymphoid tumors treated with monoclonal anti-idiotype antibodies correlation with clinical responses. Blood 69: 199-210.

19. Lopez-Diaz de Cerio A, Zabalegui N, Rodriguez-Calvillo M, Inoges S, Bendan M (2007) Anti-idiotype antibodies in cancer treatment. Oncogene 26: 35943602.

20. Losman MJ, Leung SO, Shih LB, Shevitz J, Shukla R, et al. (1995) Development and evaluation of the specificity of a rat monoclonal anti-idiotype antibody WN, to an anti-B-cell lymphoma monoclonal antibody, LL2. Cancer Res 55 5978s-5982s.

21. Monestier M, Debbas ME, Goldenberg DM (1989) Syngenic anti-idiotype monoclonal antibodies to murine anti-carcinoembryonic antigen monoclonal antibodies. Cancer Res 49: 123-126.

22. Losman MJ, Novick KE, Goldenberg DM, Monestier M (1994) Mimicry of a carcinoembryonic antigen epitope by a rat monoclonal anti-idiotype antibody. Int J Cancer 56: 580-584.

23. Van Heyningen V, Brook DJH, Van Heyningen S (1983) A simple method for ranking the affinities of monoclonal antibodies. J Immunol Methods 62: 147153.

24. Finn OJ (2008) Cancer immunotherapy. N Engl J Med 358: 2704-2715.

25. Zou W (2006) Regulatory T cells, tumor immunity and immunotherapy. Nat Rev Immunol 6: 295-307.

26. Parmiani G, Russo V, Marrari A, Gutolo G, Casati C, et al. (2007) Universal and stemness-related tumor antigens: Potential use in immunotherapy. Clin Cancer Res 13: 5675-5679.

27. Schlom J, Arlen PM, Gulley JL (2007) Cancer vaccines: moving beyond current paradigms. Clin Cancer Res 13: 3776-3782.

28. López-Díaz de Cerio A, Zabalegui N, Roderíguez M, Inogeś S, Bendandi M (2007) Anti-idiotype antibodies in cancer treatment. Oncogene 26: 3594-3602.

29. Parmiani G, Defilipo A, Novellino L, Castelli C (2007) Unique human tumo antigens: Immunobiology and use in clinical trials. J Immunol 178: 1975-1979.

30. Kantoff PW, Higano CS, Shou ND, Berger ER, Small EJ, et al. (2010) Sipuleucel-T immunotherapy for castratian resistant prostate cancer. New Eng J Med 363: 411-422.

31. Rosenberg SA, Yang TC, Restifo NS (2004) Cancer immunotherapy: moving beyond current vaccines. Nat Med 10: 909-915.

32. Davis TA, Maloney DG, Czerwinski DK, Liles TM, Levy R (1998) Anti-idiotype antibodies can induce long-term complete remissions in non-Hodgkin's lymphoma without eradicating the malignant clone. Blood 92: 1184-1190. 
Citation: Lee G, Cheung AP, Ge B, Zhu M, Li PP, et al. (2010) Monoclonal Anti-idiotype Antibodies against Carbohydrate-associate Epitope for AntiCancer Vaccine Development. J Vaccines Vaccin 1: 106. doi:10.4172/2157-7560.1000106

Page 7 of 7

33. Mittelman A, Chen ZJ, Liu CC, Hirai S, Ferrone S (1994) Kinetics of the immune response and regression of metastatic lesions following development of humoral anti-HMW-MAA immunity in three patients with advanced malignant melanoma immunized with mouse anti-idiotypic Mab MK2-23. Cancer Res 54: 415-421.

34. Mittelman A, Chen ZJ, Liu CC, Hirai S, Ferrone S, et al. (1995) Human high molecular weight-melanoma-associated antigen mimicry by mouse antiidiotypic monoclonal antibody MK2-23: modulcation of the immunogenicity in patients with malignant melanoma. Clin Cancer Res 1: 705-713.
35. Foon KA, John WJ, Chakraborty M, Das R, Teitelbaum A, et al. (1999) Clinical and immune responses in selected colon cancer patients treated with antiidiotype monoclonal antibody vaccine that mimic carcinoembryonic antigen. $J$ Clin Oncol 17: 2889-2895.

36. Sabbatini P, Dupont J, Aghajanian C, Derosa F, Poynor E, et al. (2006) Phase I study of abagovomab in patients with epithelial ovarian, fallopian tube or primary peritoneal cancer. Clin Cancer Res 12: 5503-5510. 